\title{
ON NONNEGATIVE MATRICES WITH GIVEN ROW AND COLUMN SUMS*
}

\author{
S.W. DRURY ${ }^{\dagger}$, J.K. MERIKOSKI $\ddagger$, V. LAAKSO ${ }^{\ddagger}$, AND T. TOSSAVAINEN ${ }^{\S}$
}

\begin{abstract}
Let $\mathbf{A}$ be a nonnegative $n \times n$ matrix with row sums $r_{1}, \ldots, r_{n}$ and column sums $c_{1}, \ldots, c_{n}$. Order them decreasingly: $r_{1}^{\downarrow} \geq \ldots \geq r_{n}^{\downarrow}$ and $c_{1}^{\downarrow} \geq \ldots \geq c_{n}^{\downarrow}$. The conjectures su $\mathbf{A}^{m} \leq$ $\left(r_{1} c_{1}\right)^{\frac{m}{2}}+\cdots+\left(r_{n} c_{n}\right)^{\frac{m}{2}}$ and su $\mathbf{A}^{m} \leq\left(r_{1}^{\downarrow} c_{1}^{\downarrow}\right)^{\frac{m}{2}}+\cdots+\left(r_{n}^{\downarrow} c_{n}^{\downarrow}\right)^{\frac{m}{2}}$ are considered, where su B denotes the sum of the entries of a matrix $\mathbf{B}$ and $m$ is a nonnegative integer.
\end{abstract}

Key words. Nonnegative matrix, Row sum, Column sum, Entry sum, Matrix power.

AMS subject classifications. 15A48, 15A45.

1. Introduction. Throughout this paper, $m$ and $n$ denote integers, and $\mathbf{A}=$ $\left(a_{j k}\right)$ denotes a nonnegative $n \times n$ matrix $(n \geq 1)$ with row sums $r_{1}, \ldots, r_{n}$ and column sums $c_{1}, \ldots, c_{n}$, ordered decreasingly as $r_{1}^{\downarrow} \geq \ldots \geq r_{n}^{\downarrow}$ and $c_{1}^{\downarrow} \geq \ldots \geq c_{n}^{\downarrow}$.

London [5] conjectured in 1966 and Hoffman [2] proved in 1967 that for A symmetric

$$
\operatorname{su} \mathbf{A}^{m} \leq r_{1}^{m}+\cdots+r_{n}^{m}
$$

where su $\mathbf{B}$ denotes the sum of the entries of a matrix $\mathbf{B}$ and $m \geq 0$ (define $\left.0^{0}=1\right)$. Hoffman's proof was based on certain properties of stochastic matrices. Much later, in 1985, Sidorenko [9], without knowing Hoffman's work, gave an independent proof as an elementary application of Hölder's inequality.

In 1990, Virtanen [10] generalized (1.1) to the nonsymmetric case. He proved, using majorization, that

$$
\operatorname{su} \mathbf{A}^{m} \leq \frac{1}{2}\left(\sum_{j=1}^{n} r_{j}^{m}+\sum_{j=1}^{n} c_{j}^{m}\right) .
$$

In 1991, Merikoski and Virtanen [7] gave an elementary proof for this. In 1995, they [8] proved a sharper inequality

$$
\operatorname{su} \mathbf{A}^{m} \leq\left(\sum_{j=1}^{n} r_{j}^{m}\right)^{\frac{1}{2}}\left(\sum_{j=1}^{n} c_{j}^{m}\right)^{\frac{1}{2}}
$$

${ }^{*}$ Received by the editors on 25 August 2003. Accepted for publication on 23 November 2003. Handling Editor: Hans Schneider.

${ }^{\dagger}$ Department of Mathematics and Statistics, McGill University, 805 Sherbrooke Street West, Montreal, Canada H3A 2K6 (drury@math.mcgill.ca).

${ }_{\ddagger}^{\ddagger}$ Department of Mathematics, Statistics and Philosophy, FIN-33014 University of Tampere, Finland.

$\S$ Department of Teacher Education, University of Joensuu, P.O. Box 55, FIN-57101 Savonlinna, Finland. 
and asked whether the still sharper inequality

$$
\operatorname{su} \mathbf{A}^{m} \leq\left(r_{1} c_{1}\right)^{\frac{m}{2}}+\cdots+\left(r_{n} c_{n}\right)^{\frac{m}{2}}
$$

holds.

For $m=2,(1.3)$ is actually an equality. For $m=1$ and $m=3$, Merikoski and Virtanen [8] gave counterexamples $(n \geq 2)$ but claimed erroneously that the counterexample for $m=3$ works for all $m \geq 3$. Laakso [4] noted this error and ([4], Theorem 3.2) gave a complete description about the validity of (1.3) as follows:

TheOrem 1.1. Conjecture (1.3) is

- true (equality) if $n=1$,

- true (equality) if $m=0$ or $m=2$,

- true (inequality) if $n=2$ and $m \geq 4$ is even,

- false in the remaining cases.

Merikoski and Virtanen [8] posed also the conjecture

$$
\operatorname{su} \mathbf{A}^{m} \leq\left(r_{1}^{\downarrow} c_{1}^{\downarrow}\right)^{\frac{m}{2}}+\cdots+\left(r_{n}^{\downarrow} c_{n}^{\downarrow}\right)^{\frac{m}{2}},
$$

which is stronger than (1.2) but weaker than (1.3). We will study this conjecture.

To find counterexamples, we have made heavy use of the algorithm for the transportation problem presented in Hoffman [1]. This enables one to compute numerically the maximum value of $\mathbf{x}^{T} \mathbf{A y}$ where $\mathbf{x}$ and $\mathbf{y}$ are fixed vectors and $\mathbf{A}$ runs through all nonnegative matrices whose row sum vector and column sum vector are fixed.

In Section 2 we present counterexamples to disprove (1.4) for $m=3, n \geq 8$ and $m=4, n \geq 50$. More generally, we will in Section 3 disprove this conjecture for all $m \geq 3$ if $n$ (depending on $m$ ) is large enough. Our key idea is to study the continuous analogue of this problem, which leads us to consider integral kernels. Next, in Section 4, we will prove (1.3) for all $n$ if $m$ (depending on $n$ ) is large enough.

At the end of the paper we will study the case $n=2$. Laakso's [4] proof for $n=$ $2, m$ even is extremely tedious, using brute combinatorics. We will in Section 5 give another proof, which is shorter but, regarding the easy-looking claim, still surprisingly involved. Since Laakso disproved (1.3) for $n=2, m$ odd, it is natural to ask whether (1.4) is true in this case. We will finally show that the answer is yes.

2. The cases $m=3, n \geq 8$ and $m=4, n \geq 50$. Let $p=q=n$ and denote $\mathbb{1}=(1, \ldots, 1)^{T}$. The row sum vector of $\mathbf{A}$ is $\mathbf{r}=\mathbf{A} \mathbb{1}$ and the column sum vector is $\mathbf{c}=\mathbf{A}^{T} \mathbb{1}$. Consider su $\mathbf{A}^{3}=\mathbb{1}^{T} \mathbf{A}^{3} \mathbb{1}=\mathbf{c}^{T} \mathbf{A r}$. So the optimal upper bound for su $\mathbf{A}^{3}$ is given (at least numerically) by the solution of the transportation problem [1].

Even the case $n=2$ is quite complicated. Figure 1 shows the maximum of su $\mathbf{A}^{3}$ if su $\mathbf{A}=1, \mathbf{r}=(x, 1-x)$, and $\mathbf{c}=(y, 1-y)$. Note that the contours fail to be smooth across the lines $x=y$ and $x+y=1$.

Later results will allow us to find precise upper bounds for su $\mathbf{A}^{m}$ for all $m \geq 3$ at least in the case $n=2$.

Now we disprove (1.4) for certain $m$ and $n$. 


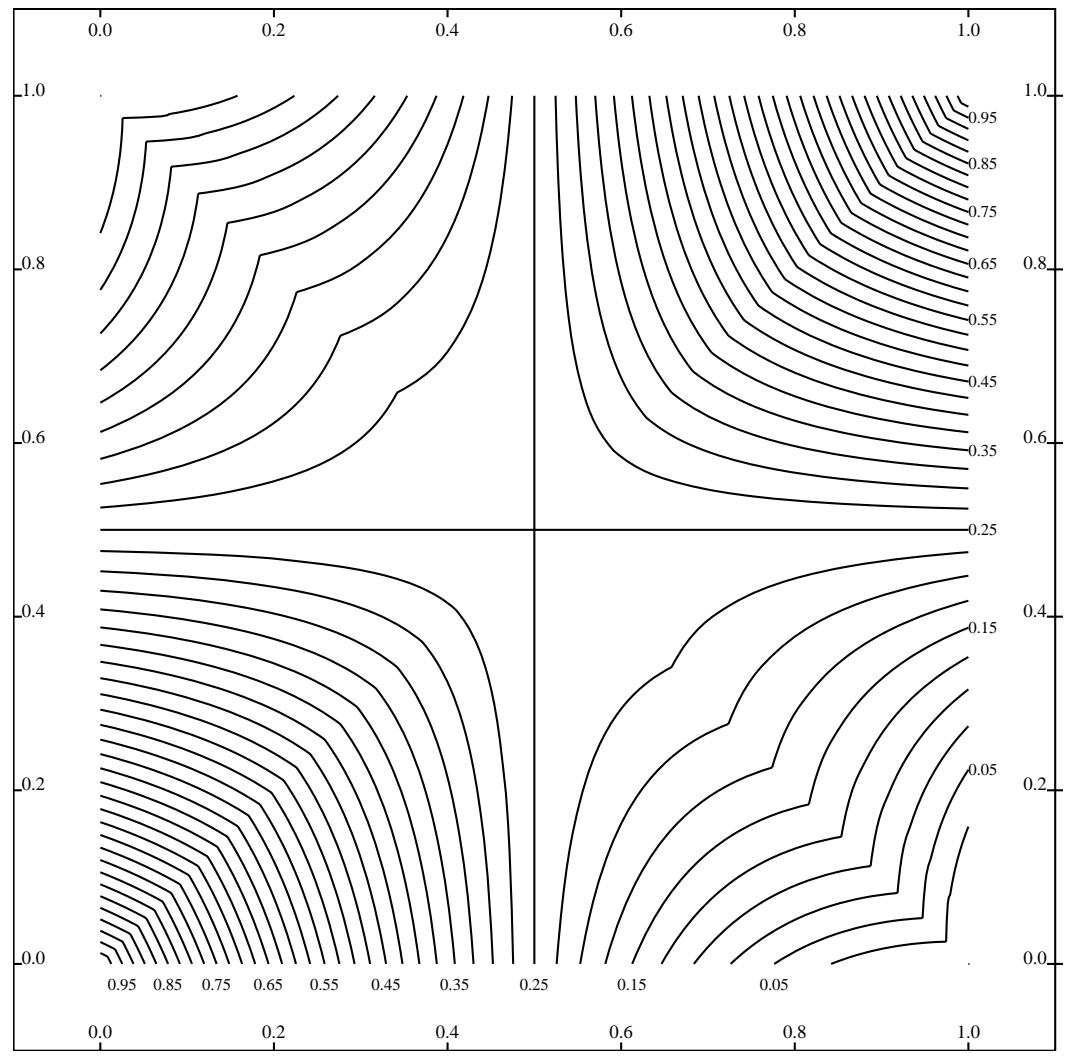

Fig. 2.1. Contour plot of $\max$ su $\mathbf{A}^{3}$ for $n=2$.

TheOREM 2.1. Conjecture (1.4) is false if $m=3$ and $n \geq 8$.

Proof. Let $\mathbf{A}$ be the $8 \times 8$ matrix with $a_{11}=5, a_{21}=1, a_{32}=\cdots=a_{82}=1$ and all the other entries zero. Then $r_{1}=5, r_{2}=\cdots=r_{8}=1, c_{1}=c_{2}=6$, $c_{3}=\cdots=c_{8}=0$, and so

$$
\mathrm{su} \mathbf{A}^{3}=180>\sum_{j=1}^{n}\left(r_{j}^{\downarrow} c_{j}^{\downarrow}\right)^{\frac{3}{2}}=30^{\frac{3}{2}}+6^{\frac{3}{2}} \approx 179.0137057 . \mathrm{\square}
$$

TheOrem 2.2. Conjecture (1.4) is false if $m=4$ and $n \geq 50$.

Proof. See the appendix. $\square$

The cases $m=3,3 \leq n \leq 7$ and $m=4,3 \leq n \leq 49$ remain open. (The case $n=2$ is discussed in Section 5.)

3. The case $m \geq 3, n$ large. Theorems 2.1 and 2.2 suggest that (1.4) is false for all $m \geq 3$ if $n$ is large enough. This indeed holds.

THEOREM 3.1. Conjecture (1.4) is false for all $m \geq 3$ if $n$ (depending on $m$ ) is large enough. 
If (1.4) were true for a fixed $m \geq 3$ and all $n \geq 1$, then a corresponding result for integral kernels would hold. This could further be extended to certain types of singular kernels given by a measure. The measure of interest is given by $d \mu(x)=x^{-\alpha} d x$ on the curve $y=x^{\beta}$ in $[1, \infty) \times[1, \infty)$ for suitable $\alpha$ and $\beta$. Here the calculations are easy, but the limiting arguments are not entirely straightforward, and it seems better to give an example in terms of matrices.

Proof of Theorem 3.1. We index our matrices from 0 to $n-1$. Let $0<s<n$, $\alpha>0$, and $0<\beta<1$. We impose the constraint $\alpha+\beta>1$. Let $x_{j}=1+\frac{s j}{n}$. Define the mapping $\nu$ by

$$
\nu(j)=\left\lfloor\frac{n}{s}\left(x_{j}^{\beta}-1\right)\right\rfloor .
$$

Then $0 \leq \nu(j) \leq j<n$ for $j=0, \ldots, n-1$. We define the $n \times n$ matrix $\mathbf{A}$ by

$$
a_{j k}= \begin{cases}x_{j}^{-\alpha} & \text { if } k=\nu(j) \\ 0 & \text { otherwise. }\end{cases}
$$

Then $r_{j}=x_{j}^{-\alpha}$ and $c_{k}=\sum_{\nu(j)=k} x_{j}^{-\alpha}$. Observe that $x_{\nu(j)} \leq x_{j}^{\beta}$ and therefore

(3.1) $\operatorname{su} \mathbf{A}^{m}=\sum_{j=0}^{n-1} x_{j}^{-\alpha} x_{\nu(j)}^{-\alpha} \cdots x_{\nu^{m-1}(j)}^{-\alpha} \geq \sum_{j=0}^{n-1} x_{j}^{-\alpha} x_{j}^{-\alpha \beta} \cdots x_{j}^{-\alpha \beta^{m-1}}=\sum_{j=0}^{n-1} x_{j}^{-\gamma}$ where $\gamma=\alpha \frac{1-\beta^{m}}{1-\beta}$.

We find an upper bound for $c_{k}$.

First assume $k \geq 1$. Let $j_{k}$ be the smallest $j$ such that $\nu(j)=k$. Then

$$
\begin{gathered}
c_{k}=\sum_{j=j_{k}}^{j_{k+1}-1}\left(1+\frac{s j}{n}\right)^{-\alpha} \leq \int_{j_{k}-1}^{j_{k+1}-1}\left(1+\frac{s x}{n}\right)^{-\alpha} d x= \\
(3.2) \beta^{-1} \int_{y_{k}}^{y_{k+1}}\left(1+\frac{s y}{n}\right)^{-\frac{\alpha}{\beta}}\left(1+\frac{s y}{n}\right)^{\frac{1}{\beta}-1} d y=\beta^{-1} \int_{y_{k}}^{y_{k+1}}\left(1+\frac{s y}{n}\right)^{-\frac{\alpha+\beta-1}{\beta}} d y,
\end{gathered}
$$

after the change of variable $1+\frac{s y}{n}=\left(1+\frac{s x}{n}\right)^{\beta}$ where $y_{k}$ is defined by

$$
1+\frac{s y_{k}}{n}=\left(1+\frac{s\left(j_{k}-1\right)}{n}\right)^{\beta}
$$

Now $\left\lfloor y_{k}\right\rfloor=\nu\left(j_{k}-1\right)=k-1$ and $\left\lfloor y_{k+1}\right\rfloor=k$, and so $y_{k+1}-y_{k} \leq 2$. Hence, by (3.2),

$$
c_{k} \leq 2 \beta^{-1}\left(1+\frac{s(k-1)}{n}\right)^{-\frac{\alpha+\beta-1}{\beta}} .
$$


For $k=0$ we proceed slightly differently

$$
\begin{aligned}
c_{0} & =\sum_{j=0}^{j_{1}-1}\left(1+\frac{s j}{n}\right)^{-\alpha} \leq 1+\sum_{j=1}^{j_{1}-1}\left(1+\frac{s j}{n}\right)^{-\alpha} \\
& \leq 1+\int_{0}^{j_{1}-1}\left(1+\frac{s x}{n}\right)^{-\alpha} d x=1+\beta^{-1} \int_{0}^{y_{1}}\left(1+\frac{s y}{n}\right)^{-\frac{\alpha+\beta-1}{\beta}} d y \\
& \leq 1+\beta^{-1}
\end{aligned}
$$

since $y_{1} \leq 1$. Note that

$$
1+\beta^{-1}<2 \beta^{-1}<2 \beta^{-1}\left(1-\frac{s}{n}\right)^{-\frac{\alpha+\beta-1}{\beta}}
$$

and so (3.3) holds also for $k=0$.

Since $\alpha+\beta>1$, the right-hand side of (3.3) decreases in $k$. Thus, finally

$$
\sum_{j=0}^{n-1}\left(r_{j}^{\downarrow} c_{j}^{\downarrow}\right)^{\frac{m}{2}} \leq\left(\frac{2}{\beta}\right)^{\frac{m}{2}} \sum_{j=0}^{n-1}\left(1+\frac{s j}{n}\right)^{-\frac{\alpha m}{2}}\left(1+\frac{s(j-1)}{n}\right)^{-\frac{m(\alpha+\beta-1)}{2 \beta}},
$$

which, together with (3.1), implies

$$
\liminf _{n \rightarrow \infty} \frac{\operatorname{su} \mathbf{A}^{m}}{\sum_{j=0}^{n-1}\left(r_{j}^{\downarrow} c_{j}^{\downarrow}\right)^{\frac{m}{2}}} \geq\left(\frac{\beta}{2}\right)^{\frac{m}{2}} \frac{\int_{1}^{1+s} x^{-\gamma} d x}{\int_{1}^{1+s} x^{-\theta} d x}
$$

where $\theta=\frac{m(\alpha \beta+\alpha+\beta-1)}{2 \beta}$. We will show that $\alpha$ and $\beta$ can be chosen so that $\gamma<1<\theta$, thus forcing a counterexample to (1.4) with $n$ and $s$ large.

We need to choose $\alpha$ so that $\frac{m-(m-2) \beta}{m(1+\beta)}<\alpha<\frac{1-\beta}{1-\beta^{m}}$. In fact, the upper and lower bounds have double contact at $\beta=1, \alpha=m^{-1}$. We have

$$
m(1+\beta)(1-\beta)-\left(1-\beta^{m}\right)(m-(m-2) \beta)=\left(\begin{array}{c}
m \\
3
\end{array}\right)(1-\beta)^{3}+\mathcal{O}\left((1-\beta)^{4}\right),
$$

showing that suitable $\alpha$ and $\beta$ exist with $\beta$ slightly smaller than 1 . $\square$

4. The case $m$ large. If $m \geq 3$ is fixed, we saw above that (1.4) is false if $n$ is large enough. If instead, we fix $n$ and make $m$ large enough, then we find the opposite result. Then (1.4) is true, and the stronger (1.3) is in "almost all" cases true. Before showing this, we recall that the Perron root of $\mathbf{A}$, denoted by $\rho(\mathbf{A})=\rho$, satisfies (see e.g., [6], p. 151)

$$
\rho \leq \max _{j}\left(r_{j} c_{j}\right)^{\frac{1}{2}} \leq\left(r_{1}^{\downarrow} c_{1}^{\downarrow}\right)^{\frac{1}{2}}
$$




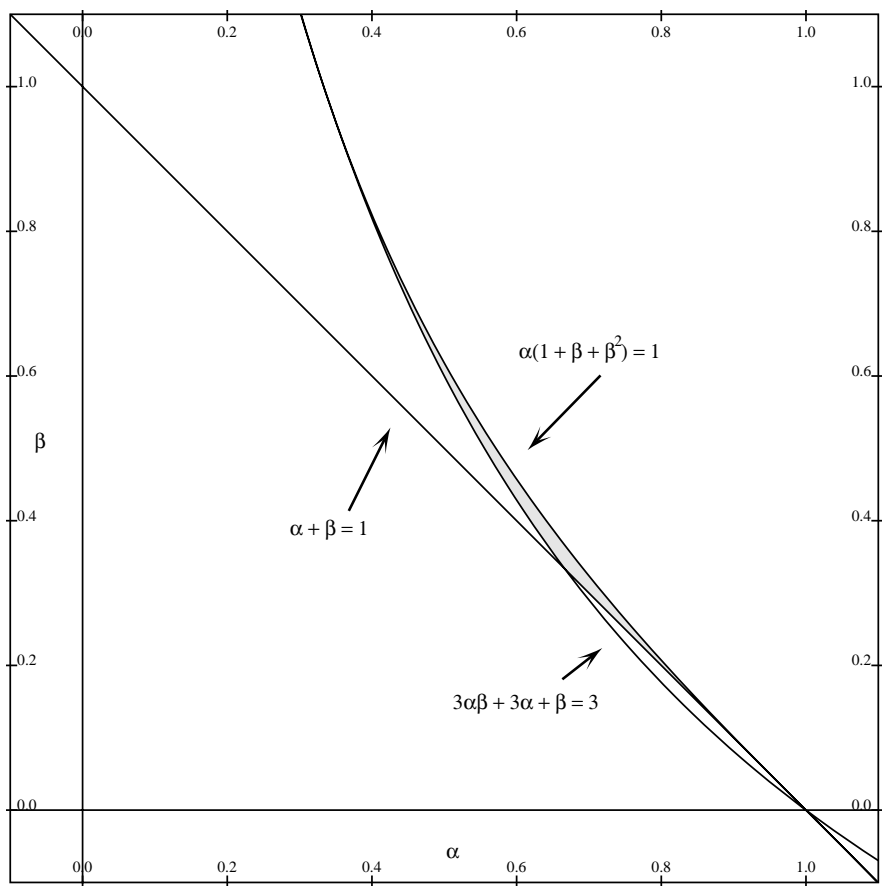

FIG. 3.1. Constraints on $\alpha$ and $\beta$ for $m=3$. The point $(\alpha, \beta)$ must be chosen in the shaded region.

TheOREM 4.1. Assume that $\rho<\max _{j}\left(r_{j} c_{j}\right)^{\frac{1}{2}}$. Then (1.3) is true for all $n \geq 1$ if $m$ (depending on $n$ ) is large enough.

Proof. Applying the norm properties of su $\mathbf{A}=\mathrm{su}|\mathbf{A}|$ (see e.g., [3], Corollary 5.6.14), we obtain

$$
\lim _{m \rightarrow \infty}\left(\operatorname{su} \mathbf{A}^{m}\right)^{\frac{1}{m}}=\rho<\max _{j}\left(r_{j} c_{j}\right)^{\frac{1}{2}}=\lim _{m \rightarrow \infty}\left(\left(r_{1} c_{1}\right)^{\frac{m}{2}}+\cdots+\left(r_{n} c_{n}\right)^{\frac{m}{2}}\right)^{\frac{1}{m}},
$$

and the claim follows. $\square$

To show that the assumption $\rho<\max _{j}\left(r_{j} c_{j}\right)^{\frac{1}{2}}$ is crucial, consider

$$
\mathbf{A}=\left(\begin{array}{lll}
0 & 4 & 0 \\
0 & 0 & 1 \\
1 & 0 & 1
\end{array}\right)
$$

which has $\rho(\mathbf{A})=2$. It can be proved by induction (for details, see [4], Example $3.3)$ that the row sums of $\mathbf{A}^{m}(m \geq 4)$, denoted by $r_{1}^{(m)}, r_{2}^{(m)}$ and $r_{3}^{(m)}$, satisfy $r_{1}^{(m)}+r_{2}^{(m)}>2.4 \cdot 2^{m}$ and $r_{3}^{(m)}>0.6 \cdot 2^{m}$. Hence

$$
\mathrm{su} \mathbf{A}^{m}=r_{1}^{(m)}+r_{2}^{(m)}+r_{3}^{(m)}>3 \cdot 2^{m},
$$


but $r_{1} c_{1}=r_{2} c_{2}=r_{3} c_{3}=4$, which implies

$$
\left(r_{1} c_{1}\right)^{m / 2}+\left(r_{2} c_{2}\right)^{m / 2}+\left(r_{3} c_{3}\right)^{m / 2}=3 \cdot 2^{m},
$$

contradicting (1.3).

THEOREM 4.2. Conjecture (1.4) is true for all $n \geq 1$ if $m$ (depending on $n$ ) is large enough.

Proof. If $\rho<\left(r_{1}^{\downarrow} c_{1}^{\downarrow}\right)^{\frac{1}{2}}$, a trivial modification of the proof of Theorem 4.1 applies. Thus, by (4.1), the case $\rho=\left(r_{1}^{\downarrow} c_{1}^{\downarrow}\right)^{\frac{1}{2}}$ remains.

Assume first that $\mathbf{A}$ is positive. Then $\mathbf{B}=\mathbf{A} \otimes \mathbf{A}^{T}$ is positive with row sums $r_{1} c_{1}, \ldots, r_{1} c_{n}, \ldots, r_{n} c_{1}, \ldots, r_{n} c_{n}$. The largest row sum of $\mathbf{B}$ is

$$
\max _{j, k} r_{j} c_{k}=r_{1}^{\downarrow} c_{1}^{\downarrow}=\rho^{2}=\rho(\mathbf{B}) .
$$

Therefore all the row sums of $\mathbf{B}$ are equal (see e.g. [6], Section 3.1.1), and so $r_{1}=\cdots=$ $r_{n}=c_{1}=\cdots=c_{n}$, which implies (1.4). Finally, we omit the positivity assumption by continuity argument.

5. The case $n=2$. We present for the main part of Theorem 1.1 a new proof, which is shorter than Laakso's [4].

TheOREM 5.1. Conjecture (1.3) is true if $n=2$ and $m \geq 0$ is even.

Lemma 5.2. For $n \geq 2$, let $\kappa_{1}>\ldots>\kappa_{n}>0$ and $t_{1}>\ldots>t_{n-1} \geq 0$, and suppose that $a_{1}, \ldots, a_{n}$ are real numbers with $a_{1}>0$. If the function

$$
f(t)=\sum_{j=1}^{n} a_{j} \kappa_{j}^{t}
$$

satisfies $f\left(t_{1}\right)=\cdots=f\left(t_{n-1}\right)=0$, then $f(t)>0$ for all $t>t_{1}$.

To prove this lemma, we apply induction.

First consider $n=2$. We can without loss of generality assume $\kappa_{2}=1$. Then $f(t)=a_{1} \kappa_{1}^{t}+a_{2}$ is strictly increasing, which proves the assertion in this case.

We next suppose that the assertion holds for $n(\geq 2)$ and claim that it holds for $n+1$. Again we can without loss of generality assume $\kappa_{n+1}=1$. Then

$$
f(t)=\sum_{j=1}^{n} a_{j} \kappa_{j}^{t}+a_{n+1}
$$

and

$$
f^{\prime}(t)=\sum_{j=1}^{n} a_{j} \kappa_{j}^{t} \ln \kappa_{j} \equiv \sum_{j=1}^{n} b_{j} \kappa_{j}^{t}
$$

where $b_{1}>0$. Applying the induction hypothesis to $f^{\prime}$, we see that (i) there are at most $n-1$ numbers $\tau_{1}>\ldots>\tau_{k} \geq 0$ such that $f^{\prime}\left(\tau_{1}\right)=\ldots=f^{\prime}\left(\tau_{k}\right)=0$, and 
(ii) if there are $n-1$ such numbers, then $f^{\prime}(t)>0$ for all $t>\tau_{1}$. But (i) implies, by Rolle's theorem, that there are at most $n$ numbers $t_{1}>\ldots>t_{k} \geq 0$ such that $f\left(t_{1}\right)=\ldots=f\left(t_{k}\right)=0$. Now assume that there are $n$ such numbers, and let $t>t_{1}$. Then $f$ is strictly increasing, since $t_{1}>\tau_{1}$ and, by (ii), $f^{\prime}(t)>0$ for $t>\tau_{1}$. Therefore $f(t)>0$, which completes the proof.

Proof of Theorem 5.1. We can without loss of generality assume that $\mathrm{su} \mathbf{A}=1$ and $r_{1}=\max \left(r_{1}, r_{2}, c_{1}, c_{2}\right)$. If the maximum is attained at $r_{2}$, then interchange both the rows and columns. If it is attained at $c_{1}$, then transpose $\mathbf{A}$. If it is attained at $c_{2}$, then interchange both the rows and columns and transpose $\mathbf{A}$.

Given row and column sums, one entry of $\mathbf{A}$ determines all the others, and so the $\mathbf{A}$ 's form a one parameter family. In fact $\mathbf{A}$ is a convex combination of

$$
\mathbf{B}=\left(\begin{array}{cc}
r+c-1 & 1-c \\
1-r & 0
\end{array}\right) \quad \text { and } \quad \mathbf{C}=\left(\begin{array}{cc}
c & r-c \\
0 & 1-r
\end{array}\right)
$$

where $r=r_{1}, 1-r=r_{2}, c=c_{1}, 1-c=c_{2}$.

The case $r=1$ reduces to $c^{m}+m c^{m-1}(1-c) \leq c^{\frac{m}{2}}$ which is easily checked, and so we assume $r<1$. Then

$$
\mathbf{A}=t\left(\begin{array}{cc}
r+c-1 & 1-c \\
1-r & 0
\end{array}\right)+(1-t)\left(\begin{array}{cc}
c & r-c \\
0 & 1-r
\end{array}\right)
$$

with $0 \leq t \leq 1$. We prefer to work with $s=\frac{1}{2}(r-c)+(1-r) t$ satisfying $\frac{1}{2}(r-c) \leq$ $s \leq \frac{1}{2}(2-r-c)$ since the eigenvalues of $\mathbf{A}$ are then $\lambda_{ \pm}=\frac{1}{2}-s \pm \sqrt{s^{2}+q}$ where $q=\left(r-\frac{1}{2}\right)\left(c-\frac{1}{2}\right)$.

For $m \geq 0$, consider

$$
\sigma_{m} \equiv \operatorname{su} \mathbf{A}^{m}=\left(1+\frac{s}{\sqrt{s^{2}+q}}\right) \lambda_{+}^{m}+\left(1-\frac{s}{\sqrt{s^{2}+q}}\right) \lambda_{-}^{m} .
$$

Omitting the trivial case $m=0$, let $m \geq 2$. The quantity

$$
\begin{aligned}
\frac{\partial \sigma_{m}}{\partial s} & =-\frac{m q}{s^{2}+q}\left(\lambda_{+}^{m-1}+\lambda_{-}^{m-1}\right)+\frac{q}{\left(s^{2}+q\right)^{\frac{3}{2}}}\left(\lambda_{+}^{m}-\lambda_{-}^{m}\right) \\
& =-q \beta^{-3}\left(m \beta\left((\alpha+\beta)^{m-1}+(\alpha-\beta)^{m-1}\right)-(\alpha+\beta)^{m}+(\alpha-\beta)^{m}\right),
\end{aligned}
$$

where $\alpha=\frac{1}{2}-s$ and $\beta=\sqrt{s^{2}+q}$, is opposite in sign to $q$. For, denoting $\gamma=\alpha \beta^{-1} \geq$ 0 , we have

$$
\begin{array}{r}
m\left((\gamma+1)^{m-1}+(\gamma-1)^{m-1}\right)-(\gamma+1)^{m}+(\gamma-1)^{m}= \\
2 \sum_{\substack{m-k \text { odd } \\
0 \leq k \leq m-1}}\left(m\left(\begin{array}{c}
m-1 \\
k
\end{array}\right)-\left(\begin{array}{c}
m \\
k
\end{array}\right)\right) \gamma^{k} \geq 0 .
\end{array}
$$


Now, since $r \geq \frac{1}{2}$, it remains to establish (1.3) for $\mathbf{B}$ if $c \leq \frac{1}{2}$ and for $\mathbf{C}$ if $c \geq \frac{1}{2}$. We first dispose of the case $q=\left(r-\frac{1}{2}\right)\left(c-\frac{1}{2}\right)=0$. Then $\sigma_{m}=\left(\frac{1}{2}\right)^{m-1}$. Because the average of $r c$ and $(1-r)(1-c)$ is $q+\frac{1}{4}=\frac{1}{4}$ and the function $x \mapsto x^{\frac{m}{2}}(x \geq 0)$ is convex, we have

$$
\frac{1}{2} \sigma_{m}=\left(\frac{1}{2}\right)^{m}=\left(\frac{1}{4}\right)^{\frac{m}{2}} \leq \frac{1}{2}\left((r c)^{\frac{m}{2}}+((1-r)(1-c))^{\frac{m}{2}}\right),
$$

which proves (1.3).

Case $\mathbf{A}=\mathbf{C}, \frac{1}{2}<c \leq r<1$. We have $t=0, s=\frac{1}{2}(r-c), \lambda_{+}=c, \lambda_{-}=1-r$. If $c=r$, then equality holds in (1.3). So we can assume $\frac{1}{2}<c<r<1$. Denoting $\mu_{ \pm}=\frac{1}{2} \pm \sqrt{q}$, we obtain

$$
\sigma_{m}=\frac{2 r-1}{r+c-1} c^{m}+\frac{2 c-1}{r+c-1}(1-r)^{m} \leq \mu_{+}^{m}+\mu_{-}^{m}
$$

by Lemma 5.2 , because equality holds in (5.2) for $m=0,1$ and 2, and because $0<1-r<\mu_{-}<c<\mu_{+}$. Finally, we note that

$$
\mu_{+}^{m}+\mu_{-}^{m} \leq(r c)^{\frac{m}{2}}+((1-r)(1-c))^{\frac{m}{2}},
$$

due to the inequalities $(1-r)(1-c) \leq \mu_{-}^{2} \leq \mu_{+}^{2} \leq r c$, the convexity of the function $x \mapsto x^{\frac{m}{2}}(x \geq 0)$, and the equality $\mu_{+}^{2}+\mu_{-}^{2}=r c+(1-r)(1-c)$.

Now (5.2) and (5.3) imply (1.3).

Case $\mathbf{A}=\mathbf{B}, 0<c<\frac{1}{2}<r<1$. We have $t=1, s=\frac{1}{2}(r-c)+1-r=\frac{1}{2}(2-r-c)$. If $c=1-r$, then $\mathbf{B}^{2}=r(1-r) \mathbf{I}$, and equality in (1.3) follows. We can therefore assume $1-c<r$, and hence $s<\frac{1}{2}$. Unfortunately $\lambda_{ \pm}$do not simplify as in the previous case. We note that $\lambda_{-}<0$ and define $\nu_{ \pm}=\lambda_{ \pm}^{2}>0$. Since $m \geq 2$ is even, we set $k=m / 2 \geq 1$. By Lemma 5.2,

$$
\sigma_{2 k}=\left(1+\frac{s}{\sqrt{s^{2}+q}}\right) \nu_{+}^{k}+\left(1-\frac{s}{\sqrt{s^{2}+q}}\right) \nu_{-}^{k} \leq 2\left(q+\frac{1}{4}\right)^{k},
$$

because equality holds for $k=0$ and 1 , and because $0<\nu_{-}<\nu_{+}<q+\frac{1}{4}$.

Noting that $q+\frac{1}{4}$ is the average of $r c$ and $(1-r)(1-c)$, and applying the convexity of the function $x \mapsto x^{k}(x \geq 0)$, we finally obtain

$$
2\left(q+\frac{1}{4}\right)^{k} \leq(r c)^{k}+((1-r)(1-c))^{k} .
$$

Now (5.4) and (5.5) imply (1.3). $\square$

If $n=2$ and $m$ is odd, then (1.3) is false by Theorem 1.1, but we show that the weaker (1.4) is true.

Theorem 5.3. If $n=2$, then (1.4) is true for all $m \geq 0$. 
Proof. Since $\sum_{j=1}^{2}\left(r_{j} c_{j}\right)^{\frac{m}{2}} \leq \sum_{j=1}^{2}\left(r_{j}^{\downarrow} c_{j}^{\downarrow}\right)^{\frac{m}{2}}$, the claim follows from Theorem 5.1 if $m$ is even. We therefore assume $m$ odd and proceed as in the proof of Theorem 5.1. Again, there are two cases.

Case $\mathbf{A}=\mathbf{C}, \frac{1}{2}<c \leq r<1$. The proof is identical to the corresponding part of Theorem 5.1 , because the hypothesis $m$ even is not used there.

Case $\mathbf{A}=\mathbf{B}, 0<c<\frac{1}{2}<r<1$. Expanding $\lambda_{ \pm}^{m}$ in (5.1) we find that

$$
\begin{aligned}
\sigma_{m}=\frac{2 s}{\sqrt{s^{2}+q}} & \sum_{\substack{k=0 \\
k \text { even } \\
\text { even }}}^{m-1}\left(\begin{array}{c}
m \\
k
\end{array}\right)\left(\frac{1}{2}-s\right)^{k}\left(s^{2}+q\right)^{\frac{m-k}{2}}+ \\
& 2 \sum_{\substack{k=1 \\
k \text { odd }}}^{m}\left(\begin{array}{c}
m \\
k
\end{array}\right)\left(\frac{1}{2}-s\right)^{k}\left(s^{2}+q\right)^{\frac{m-k}{2}} .
\end{aligned}
$$

In the first sum every $m-k \geq 1$, which allows us to cancel the denominator. Since $q=\left(r-\frac{1}{2}\right)\left(c-\frac{1}{2}\right)<0$ and $0<s=1-\frac{1}{2} r-\frac{1}{2} c \leq \frac{1}{2}$, we can bound $\left(s^{2}+q\right)^{\frac{m-k}{2}} \leq s^{m-k}$. Thus, by (5.6),

$$
\begin{aligned}
\sigma_{m} & \leq 2 \sum_{k=0}^{m}\left(\begin{array}{c}
m \\
k
\end{array}\right)\left(\frac{1}{2}-s\right)^{k} s^{m-k}=2\left(\frac{1}{2}\right)^{m} \leq 2\left(\frac{1}{4}-q\right)^{\frac{m}{2}} \\
& =2\left(\frac{1}{2} r+\frac{1}{2} c-r c\right)^{\frac{m}{2}} \leq(r(1-c))^{\frac{m}{2}}+(c(1-r))^{\frac{m}{2}},
\end{aligned}
$$

and (1.4) follows.

Acknowledgment. We thank Ari Virtanen for valuable discussions and the referee for helpful suggestions.

Appendix: Counterexample for $m=4, n=50$. Let $\mathbf{A}$ be the $50 \times 50$ matrix with

$$
\begin{aligned}
& a_{11}=678, a_{21}=73, a_{22}=519, a_{32}=153, a_{33}=4, a_{43}=102, \\
& a_{53}=a_{63}=91, a_{73}=78, a_{83}=a_{93}=77, a_{10,3}=76, \\
& a_{11,3}=a_{13,4}=a_{14,4}=a_{15,4}=a_{16,4}=a_{17,4}=a_{18,4}=a_{20,5}= \\
& a_{21,5}=a_{22,5}=a_{23,5}=a_{24,5}=a_{25,5}=a_{27,6}=a_{28,6}=a_{29,6}= \\
& a_{30,6}=a_{31,6}=a_{32,6}=a_{34,7}=a_{35,7}=a_{36,7}=a_{37,7}=a_{39,8}= \\
& a_{40,8}=a_{41,8}=a_{42,8}=a_{44,9}=a_{45,9}=a_{46,9}=a_{47,9}=a_{49,10}=a_{50,10}=74, \\
& a_{12,3}=2, a_{12,4}=72, a_{19,4}=a_{38,7}=45, a_{19,5}=a_{38,8}=29, a_{26,5}=63, \\
& a_{26,6}=11, a_{33,6}=50, a_{33,7}=24, a_{43,8}=36, a_{43,9}=38, \\
& a_{48,9}=1, a_{48,10}=73,
\end{aligned}
$$


and all the other elements zero. Then

$$
\begin{aligned}
& r_{1}=678, r_{2}=592, r_{3}=157, r_{4}=102, r_{5}=r_{6}=91, r_{7}=78, \\
& r_{8}=r_{9}=77, r_{10}=76, r_{11}=\cdots=r_{50}=74
\end{aligned}
$$

and

$$
\begin{aligned}
& c_{1}=751, c_{2}=c_{3}=672, c_{4}=561, c_{5}=536, c_{6}=505, c_{7}=365, \\
& c_{8}=361, c_{9}=335, c_{10}=221, c_{11}=\cdots=c_{50}=0
\end{aligned}
$$

are in decreasing order. We have su $\mathbf{A}^{4}=440830340180$, but $\left(r_{1} c_{1}\right)^{2}+\cdots+\left(r_{50} c_{50}\right)^{2}=$ 438953283911, contradicting (1.4).

\section{REFERENCES}

[1] A.J. Hoffman. On simple linear programming problems. In Proceedings of Symposia in Pure Mathematics, Vol. VII: Convexity, Amer. Math. Soc., Providence, RI, 1963, pp. 317-327.

[2] A.J. Hoffman. Three observations on nonnegative matrices. Journal of Research of the National Bureau of Standards, 71B:39-41, 1967.

[3] R.A. Horn and C.R. Johnson. Matrix Analysis. Cambridge University Press, Cambridge, 1985.

[4] V. Laakso. Matriisin potenssin alkioiden summa. Licentiate's thesis, University of Tampere, 1999.

[5] D. London. Two inequalities in nonnegative symmetric matrices. Pacific Journal of Mathematics, 16:515-536, 1966.

[6] M. Marcus and H. Minc. A Survey of Matrix Theory and Matrix Inequalities. Dover Publications, New York, 1992.

[7] J.K. Merikoski and A. Virtanen. On the London-Hoffman inequality for the sum of elements of powers of nonnegative matrices. Linear and Multilinear Algebra, 30:257-259, 1991.

[8] J.K. Merikoski and A. Virtanen. Research Problem: An inequality for the sum of elements of powers of nonnegative matrices. Linear and Multilinear Algebra, 39:307-308, 1995.

[9] A. Sidorenko. Dokazatel'stvo predpolozhenya Londona o summakh ehlementov neotrizatel'nykh matrits. Matematicheskie Zametki, 38:376-377, 1985. English translation: Mathematical Notes, 38:716-717, 1985

[10] A. Virtanen. Schur-konveksisuudesta ja sen sovelluksista matriisiepäyhtälöihin. Licentiate's thesis, University of Tampere, 1990. 